\title{
A multi-staged neuropeptide response to traumatic brain injury
}

\author{
José Luís Alves ${ }^{1,2}\left[\right.$ ] João Mendes ${ }^{1} \cdot$ Ricardo Leitão $^{2} \cdot$ Ana Paula Silva $^{2} \cdot$ Anabela Mota Pinto $^{1}$
}

Received: 14 March 2020 / Accepted: 28 June 2020

(c) Springer-Verlag GmbH Germany, part of Springer Nature 2020

\begin{abstract}
Purpose As the most abundant neuropeptides in Central Nervous System, Substance P and Neuropeptide Y are arguably involved in the response to brain trauma. This study aims to characterize a new concept of multi-staged neuropeptide response to TBI.

Methods This study assessed Substance P, Neuropeptide Y, S100B, standard inflammatory parameters and ionic disturbance in TBI victims, with and without intracranial lesions, and healthy controls. In the group with intracranial lesions, blood samples were drawn until $6 \mathrm{~h}$ after initial trauma, at $48 \mathrm{~h}$ and 7 days post-TBI.

Results An early increase in Substance P (mean 613.463 $\pm 49.055 \mathrm{SE} 6 \mathrm{~h}$ post-TBI with brain contusions vs. $441.441 \pm 22.572$ $\mathrm{SE}$ pg/dL control group) is evident. Concerning TBI without intraparenchymatous lesions, an increase in substance $\mathrm{P}$ is also present $(825.60 \pm 23.690 \mathrm{SE} \mathrm{pg} / \mathrm{dL})$. Following an initial increase and subsequent fall in NPY levels $(45.997 \pm 4.96$ SE $6 \mathrm{~h}$ post-TBI vs. $32.395 \pm 4.056 \mathrm{SE} 48 \mathrm{~h}$ post-TBI vs. $19.700 \pm 1.462 \mathrm{SE} \mathrm{pg} / \mathrm{mL}$ control group), a late increase in NPY is obvious $(43.268 \pm 6.260 \mathrm{SE} \mathrm{pg} / \mathrm{mL} 7$ day post-TBI). Post-traumatic hypomagnesemia $(0.754 \pm 0.015 \mathrm{SE} 6 \mathrm{~h}$ post-TBI vs. $0.897 \pm 0.021 \mathrm{SE} \mathrm{mmol} / \mathrm{L}$ control group) and a peak in S100B $(95.668 \pm 14.102 \mathrm{SE} 6 \mathrm{~h}$ post-TBI vs. $30.187 \pm 3.347 \mathrm{SE} \mathrm{pg} /$ $\mathrm{mL}$ control group) are also present.

Conclusion A multi-staged neuropeptide response to TBI is obvious and represents a potential therapeutic strategy for the treatment of intraparenchymal lesions and cerebral edema following TBI.
\end{abstract}

Keywords Brain trauma $\cdot$ Neurogenic inflammation $\cdot$ Neuropeptide $Y \cdot$ Substance $P$

\section{Introduction}

Traumatic brain injury (TBI) is a major public health issue, with economic/social significance [1-3]. As a consequence of brain trauma, primary damage is followed by cellular/ biochemical deregulation, neurometabolic disturbance, hippocampal synaptic disturbance and neuronal/astrocyte degeneration (CA1/CA3 layers, Dentate Gyrus) [4, 5], related to glutamatergic excitotoxicity [6-10]. Ensuing Blood-Brain Barrier (BBB) breakdown and neurogenic inflammation $[11,12]$ will then reinforce brain edema and

José Luís Alves

jlmonteiroalves@gmail.com

1 Faculdade de Medicina da Universidade de Coimbra, Instituto de Patologia Geral, Rua Larga, no. 3, 3000-370 Coimbra, Portugal

2 Faculdade de Medicina da Universidade de Coimbra, Coimbra Institute for Clinical and Biomedical Research, Rua Larga, no. 3, 3000-370 Coimbra, Portugal early/late apoptosis [7]. Effective treatments are yet to be seen, despite recent developments in reliable biomarkers [13].

Increased post-traumatic levels of Substance P (SP) and perivascular immunoreactivity following TBI have been reported in the literature $[14,15]$. Substance $P$, which is acting on NK1 tachykinin receptors (NK1-r) [16, 17], is partially responsible for post-TBI neurogenic inflammation, promoting vasogenic edema $[18,19]$, chemokine production and adhesion molecule expression [20]. Substance P is regulated by magnesium/NMDA-receptor signaling pathways [21]. Magnesium has been reported to be neuroprotective, regulating NMDA receptors and attenuating neuronal/ astrocyte excitotoxicity. Other studies have shown a postTBI decrease in intracellular and serum magnesium levels, influenced by SP [22].

Neuropeptide Y (NPY), the most abundant brain neuropeptide and major neurotransmitter [23], modulates the cytotoxic environment following stroke or epilepsy and it is suggested to support neuronal regeneration [24, 
25]. Neuropeptide $Y$ influences neuroprotective pathways affecting glutamatergic hippocampal excitability (NPY Y2 receptors) and pro-neurogenic (NPY Y1 receptors) and promigratory activity (Dentate Gyrus, Sub-Ventricular Zone) [26-28]. Despite its ubiquitous influence, NPY's response to TBI is relatively unknown.

S100 beta (S100B), an intracellular S100-group $\mathrm{Ca}^{2+}$-binding protein [29], located primarily in astrocytes [30], is a useful TBI biomarker [31], displaying adequate sensitivity in detecting and assessing progression of brain lesions, as it is correlated with the amount of injured tissue $[32,33]$.

We hypothesize that TBI leads to a multi-staged neuropeptide response, as shown by an immediate response concerning SP, followed by compensatory NPY up-regulation. This study aimed at assessing neuropeptide response among different groups, including TBI with and without obvious brain injury, its temporal profile and relation to S100B and Magnesium levels, knowingly affected by TBI [3, 13].

\section{Materials and methods}

A prospective, single-center analysis of all consecutive patients with clinical diagnosis of TBI and and indication for head CT imaging was performed from January 2017 to July 2019 at the Hospitais Universitários de Coimbra (Coimbra's University Hospitals).

A thorough Informed Consent Form and the protocol for selection of patients, preservation of anonymity and handling of clinical data were approved by the Ethics Committee both in Centro Hospitalar e Universitário de Coimbra (Coimbra's Hospital and University Centre) and Coimbra's University.

Diagnosis of TBI was confirmed upon anamnesis, with confirmation of significant head trauma, and clinical examination. Timing of TBI with respect to clinical observation was confirmed by the patient (when appropriate), accompanying persons and/or medical teams involved in pre-hospital management. Indication for performing initial CT scan was in accordance with the Portuguese National Protocol in Traumatic Brain Injury-moderate to severe TBI, according to Glasgow Coma Scale (GCS) score; abnormal neurological examination; significant loss of consciousness; suspected fracture; known risk factors ( $>65$ year, alcoholism, drug abuse, epilepsy, coagulopathy, previous cranial surgery). CT scans (Siemens SOMATOM go-All ${ }^{\circledR}$ ) were evaluated by an independent radiologist (from a group of six dedicated neuroradiologists) and classified as showing intraparenchymal lesions (contusions, hematomas) or not.

Exclusion criteria included: pediatric patients (17 years or less); patients > 80 years; active or recent infection; acute/ chronic renal or gastrointestinal disease; inflammatory bowel disease; alcohol dependence; uncontrolled or recently diagnosed diabetes; recent vomiting/diarrhea; previous/present cranial or intracranial pathologies; concomitant cranial/ intracranial traumatic findings (fractures; subdural/epidural hematomas; subarachnoid hemorrhage); simultaneous significant traumatic findings (thoracic, abdomen, limbs, spine or others); recent traumatic injuries; patients prescribed with diuretics, angiotensin converting-enzyme inhibitors, gentamicin, amphotericin and others, interfering with magnesium metabolism. Patients initially enrolled but who posteriorly (in initial 7 days post-injury) required any tipe of surgical procedure or developed an infectious or other significant medical condition were also excluded. All previously mentioned conditions or medications can potentially interfere with inflammation pathways, neuropeptides levels or ionic balance.

Patients were enrolled into the study upon meeting inclusion/exclusion criteria and assigned to their corresponding group following assessment of corresponding head CT scan findings.

Participants were divided into five groups, $n=35$ per group (as $n>30$ is usually considered a minimum for large samples regarding Central Limit Theorem): Control group (healthy volunteers) - Group A; TBI victims without parenchymal lesions (as shown on CT scans and despite significant head trauma), $6 \mathrm{~h}$ or less after trauma-Group B; TBI victims with visible parenchymal lesions (as shown on $\mathrm{CT}$ scans), $6 \mathrm{~h}$ or less after TBI-Group $C-6 h$; TBI victims with visible parenchymal lesions, $48 \mathrm{~h}$ after TBI-Group $C-48 \mathrm{~h}$; TBI victims with visible parenchymal lesions, 7 days after TBI-Group C-7 day.

A subset of patients, initially included in group C- $6 \mathrm{~h}$, underwent repeated samplings in all different timings $(6 \mathrm{~h}$, $48 \mathrm{~h}$ and 7 days) and is, therefore, included in all groups $\mathrm{C}$.

Blood samples were collected via direct venipuncture, and immediately processed in Hospital's laboratories, as follows:

- Group A-elective blood sampling

- Group B-blood sampling until $6 \mathrm{~h}$ following TBI

- Group C-6 h-blood sampling until $6 \mathrm{~h}$ following TBI

- Group C-48 h-blood sampling $48 \mathrm{~h}$ following TBI

- Group C-7 day—blood sampling 7 days following TBI

Peripheral blood was collected to 8-mL heparin tubes (S-Monovette ${ }^{\circledR}$ ), homogenized and transferred within a 5-min interval to a $15-\mathrm{mL}$ Falcon tube containing aprotinin, preventing protein degradation (concentration $0.014 \mathrm{TIU} /$ $\mathrm{mL}$ ). After gently inverting, Falcon tubes were left to rest for $20 \mathrm{~min}$. Falcon tubes were then centrifuged for $15 \mathrm{~min}$ at $1000 \mathrm{~g}$ and $4{ }^{\circ} \mathrm{C}$. Samples were stored in $200-\mu \mathrm{L}$ aliquots at $-80{ }^{\circ} \mathrm{C}$ to prevent repetitive freeze/thaw cycles. Fifty $\mu \mathrm{L}$ of plasma was used in duplicates and absorbance was 
determined (BioRad ${ }^{\circledR}$ model 600 plate reader). Average of duplicates readings was performed and a standard curve was generated using a four-parameter logistic curve-fit to determine plasma concentrations in $\mathrm{pg} / \mathrm{mL}$.

Determination of NPY, S100B and Substance P plasma levels was performed by Enzyme-Linked Immunosorbent Assay (ELISA) [(NPY and S100B kits Merck KGaA ${ }^{\circledR}$ (New Jersey, USA); SP kits R\&D Systems ${ }^{\circledR}$ kit (Minnesota, USA)]. Dilutions for the ELISAs took place for SP (1:2, taken into consideration for final calculations). The following detection limits were used for the different assays: $16.8-43.8 \mathrm{pg} / \mathrm{mL}$ dynamic range for SP (according to the supplier); $2.7 \mathrm{pg} / \mathrm{mL}$ for $\mathrm{S} 100 \mathrm{~B} ; 2 \mathrm{pg} / \mathrm{mL}$ for NPY.

Determination of Calcium, Magnesium, Sodium, Potassium, Chloride, C-Reactive Protein (CRP) and Osmolality was undertaken. Blood samples were processed on Architect analyzers (Abbot Diagnostics ${ }^{\circledR}$ ): ionogram indirect potentiomety (Sodium, Potassium, Chloride); enzymatic assays (Magnesium); immunoturbidimetry and arsenazo III Calcium complexes assays.

\section{Statistical analysis}

All data were analyzed using IBM SPSS Statistics version 24.0 and are presented as mean \pm standard error of the mean (SEM). For comparison of parametric results between multiple groups, one-way analysis of variance (ANOVA), followed by post-hoc Tukey's test (for equal sample sizes) or Tukey-Kramer test (for unequal sample sizes) was performed. Concerning the sub-group of patients who underwent repeated measures, a non-parametric Friedman Test was performed, according to the specific data requirements and non-gaussian distribution. A $p$ value less than 0.05 $(p \leq 0.05)$ was considered statistically significant.

\section{Results}

A total of 129 patients were included in the study, distributed in five distinct groups as outlined in "Materials and methods". For each group, 35 patients (or controls, concerning group A) were enrolled as an end-point- $66.6 \%$ males in total (see Table 1 for group-specific findings). The average age was: 48.8 year $\pm 1.969 \mathrm{SE}$ in group A; 61.4 year \pm 3.206 $\mathrm{SE}$ in group $\mathrm{B} ; 65.03$ year $\pm 2.440 \mathrm{SE}$ in group $\mathrm{C}-6 \mathrm{~h}$; 65.06 year $\pm 2.477 \mathrm{SE}$ in group $\mathrm{C}-48 \mathrm{~h} ; 65.4$ year \pm 2.496 SE in group C-7 day (range, considering all groups, from 27 till 80 years). No specific findings with statistical significance were obvious concerning age or gender. Two patients (6\%) initially enrolled in group C-6 h died in the first $48 \mathrm{~h}$ following TBI. From group C- 6 h, 23 patients were carried over and included in subsequent groups $\mathrm{C}-48 \mathrm{~h}$ and C-7 day, forming a specific set of patients with consecutive sampling at $6 \mathrm{~h}, 48 \mathrm{~h}$ and 7 days following TBI (average age-63.8 year; $61 \%$ males, $n=14$ ) (Table 2).

Missed samples and exclusion of obvious outliers explain differences in group sizes concerning obtained results.

At the moment of blood sampling, the number of patients who were admitted in Neurointensive Care Unit was as follows: group C-6 h-nine patients (25.7\%); group C-48 hten patients (28.6\%); and group C-7 day-nine patients $(22.9 \%)$.

\section{NPY}

On one-way ANOVA test, there was a significant effect of TBI on NPY levels among different groups- $[F(4$, $151)=4.76, p=0.0012]$, post-hoc Tukey-Kramer method test (Fig. 1a). Significant increases in NPY levels are observed when comparing victims of TBI with brain contusions at $6 \mathrm{~h}$ and controls [group C-6 h (mean $45.997 \pm 4.968$ $\mathrm{SE}, n=32$ ) vs. group A (mean $19.702 \pm 1.462 \mathrm{SE}, n=31$ )] and when comparing victims of TBI with and without brain contusions (at $6 \mathrm{~h}$ ) [group C-6 h vs. group B (mean $29.567 \pm 5.427 \mathrm{SE}, n=29$ )]. Neuropeptide $\mathrm{Y}$ is also significantly increased when comparing TBI with brain contusions at $6 \mathrm{~h}$ and $48 \mathrm{~h}$ post-TBI [group C-6 h vs. group C-48 h (mean 32.395 $\pm 4.056 \mathrm{SE}, n=32$ )]. A significant increase is again obvious if comparing victims of TBI with brain contusions at 7 days and controls [group C-7 day (mean $43.268 \pm 6.260 \mathrm{SE}, n=30$ ) vs. group A].

Considering the subset of patients with paired samples (repeated blood sampling in the same patient at $6 \mathrm{~h}, 48 \mathrm{~h}$ and 7 days post-TBI) $(n=23)$, a similar pattern in NPY levels is displayed (Fig. 1b) - significant rise within the first $6 \mathrm{~h}$ (mean 39.924 $\pm 6.487 \mathrm{SE}$ ), with NPY levels declining out to $48 \mathrm{~h}$ (mean $28.929 \pm 4.867 \mathrm{SE}$ ) and increasing again until 7 days following TBI (mean 43.467 \pm 8.072 SE). These differences in mean values did not reach statistical significance upon non-parametric Friedman Test for repeated measures: $\chi^{2}-5.826087\left(\alpha-0.05 ; \mathrm{dF}-2 ; \chi^{2}\right.$ critical value -5.99147$)$.

In regard to NPY levels and its relation to initial GCS scores in group C-6 h, considerable differences were present (Fig. 2): mild TBI (GCS 14-15) -mean 40.114 \pm 11.435 SE, $n=13$; moderate TBI (GCS 9-13) -mean 29.460 \pm 3.950 SE, $n=10$; severe TBI (GCS 3-8)-mean 53.210 \pm 11.910 $\mathrm{SE}, n=9$. Those differences did not reach statistical significance upon non-parametric three-groups comparison with Kruskal-Wallis test by ranks: $\chi^{2}-1.180461(\alpha-0.05$; $\mathrm{dF}-2 ; \chi^{2}$ critical value -5.99147 ).

\section{Substance P}

On one-way ANOVA test, there was a significant effect of TBI on SP levels among different groups- $[F(4$, $100)=8.190, p<0.001]$, post-hoc Tukey-Kramer method 
Table 1 General view on results concerning different groups

\begin{tabular}{|c|c|c|c|c|c|}
\hline & Group A & Group B & Group C-6 h & Group C-48 h & Group C-7 day \\
\hline$N$ (initial) & 35 & 35 & 35 & 35 & 35 \\
\hline Age (mean) & $48.8 \pm 1.969 \mathrm{SE}$ & $61.4 \pm 3.206 \mathrm{SE}$ & $65.4 \pm 2.440 \mathrm{SE}$ & $65.1 \pm 2.477 \mathrm{SE}$ & $64.7 \pm 2.496 \mathrm{SE}$ \\
\hline Age $(\max )$ & 61 & 79 & 80 & 80 & 80 \\
\hline Age (min) & 26 & 27 & 27 & 27 & 27 \\
\hline Male/female & $21 / 14$ & $23 / 12$ & $24 / 11$ & $20 / 15$ & $21 / 14$ \\
\hline \multicolumn{6}{|l|}{ GCS on admission $(n)$} \\
\hline $14-15$ & 35 & 25 & 16 & 16 & 19 \\
\hline $9-13$ & & 10 & 14 & 9 & 8 \\
\hline $3-8$ & & & 5 & 10 & 8 \\
\hline Deaths ( 7 days post-TBI) & - & - & 2 & - & - \\
\hline NPY (pg/mL) & $\begin{array}{l}19.702 \pm 1.462 \mathrm{SE} \\
(n=31)\end{array}$ & $\begin{array}{l}29.567 \pm 5.427 \mathrm{SE} \\
(n=29)\end{array}$ & $\begin{array}{l}45.997 \pm 4.968 \mathrm{SE} \\
(n=32)\end{array}$ & $\begin{array}{l}32.395 \pm 4.056 \mathrm{SE} \\
(n=32)\end{array}$ & $\begin{array}{l}43.268 \pm 6.260 \mathrm{SE} \\
(n=30)\end{array}$ \\
\hline $\mathrm{SP}(\mathrm{pg} / \mathrm{mL})$ & $\begin{array}{l}441.441 \pm 22.572 \mathrm{SE} \\
(n=31)\end{array}$ & $\begin{array}{l}825.606 \pm 23.690 \mathrm{SE} \\
(n=30)\end{array}$ & $\begin{array}{l}613.463 \pm 49.055 \mathrm{SE} \\
(n=26)\end{array}$ & $\begin{array}{l}587.576 \pm 48.363 \mathrm{SE} \\
(n=26)\end{array}$ & $\begin{array}{l}620.083 \pm 46.743 \mathrm{SE} \\
(n=27)\end{array}$ \\
\hline $\mathrm{S} 100 \mathrm{~B}(\mathrm{pg} / \mathrm{mL})$ & $\begin{array}{l}30.187 \pm 3.347 \mathrm{SE} \\
(n=31)\end{array}$ & $\begin{array}{l}42.303 \pm 6.302 \mathrm{SE} \\
(n=29)\end{array}$ & $\begin{array}{l}95.668 \pm 14.102 \mathrm{SE} \\
(n=22)\end{array}$ & $\begin{array}{l}71.778 \pm 9.556 \mathrm{SE} \\
(n=23)\end{array}$ & $\begin{array}{l}58.860 \pm 13.708 \mathrm{SE} \\
(n=22)\end{array}$ \\
\hline Magnesium (mmol/L) & $\begin{array}{l}0.897 \pm 0.021 \mathrm{SE} \\
(n=35)\end{array}$ & $\begin{array}{l}0.861 \pm 0.039 \mathrm{SE} \\
(n=29)\end{array}$ & $\begin{array}{l}0.754 \pm 0.015 \mathrm{SE} \\
(n=33)\end{array}$ & $\begin{array}{l}0.811 \pm 0.019 \mathrm{SE} \\
(n=34)\end{array}$ & $\begin{array}{l}0.925 \pm 0.039 \mathrm{SE} \\
(n=34)\end{array}$ \\
\hline Calcium (mg/dL) & $\begin{array}{l}9.46 \pm 0.063 \mathrm{SE} \\
(n=35)\end{array}$ & $\begin{array}{l}9.10 \pm 0.102 \mathrm{SE} \\
(n=35)\end{array}$ & $\begin{array}{l}8.73 \pm 0.149 \mathrm{SE} \\
(n=35)\end{array}$ & $\begin{array}{l}8.63 \pm 0.098 \mathrm{SE} \\
(n=35)\end{array}$ & $\begin{array}{l}8.71 \pm 0.135 \mathrm{SE} \\
(n=35)\end{array}$ \\
\hline $\mathrm{CRP}(\mathrm{mg} / \mathrm{L})$ & $\begin{array}{l}0.461 \pm 0.244 \mathrm{SE} \\
(n=35)\end{array}$ & $\begin{array}{l}1.435 \pm 0.518 \mathrm{SE} \\
(n=35)\end{array}$ & $\begin{array}{l}1.674 \pm 0.469 \mathrm{SE} \\
(n=35)\end{array}$ & $\begin{array}{l}7.706 \pm 1.106 \mathrm{SE} \\
(n=35)\end{array}$ & $\begin{array}{l}6.348 \pm 1.244 \mathrm{SE} \\
(n=35)\end{array}$ \\
\hline Sodium (mmol/L) & $\begin{array}{l}140.06 \pm 0.415 \mathrm{SE} \\
(n=31)\end{array}$ & $\begin{array}{l}138.57 \pm 0.570 \mathrm{SE} \\
(n=31)\end{array}$ & $\begin{array}{l}137.76 \pm 0.682 \mathrm{SE} \\
(n=31)\end{array}$ & $\begin{array}{l}139.20 \pm 0.718 \mathrm{SE} \\
(n=31)\end{array}$ & $\begin{array}{l}137.53 \pm 0.816 \mathrm{SE} \\
(n=31)\end{array}$ \\
\hline Potassium (mmol/L) & $\begin{array}{l}4.55 \pm 0.101 \mathrm{SE} \\
(n=33)\end{array}$ & $\begin{array}{l}4.08 \pm 0.073 \mathrm{SE} \\
(n=34)\end{array}$ & $\begin{array}{l}4.06 \pm 0.066 \mathrm{SE} \\
(n=28)\end{array}$ & $\begin{array}{l}3.89 \pm 0.082 \mathrm{SE} \\
(n=31)\end{array}$ & $\begin{array}{l}3.94 \pm 0.105 \mathrm{SE} \\
(n=31)\end{array}$ \\
\hline Chloride (mmol/L) & $\begin{array}{l}105.19 \pm 0.338 \mathrm{SE} \\
(n=35)\end{array}$ & $\begin{array}{l}105.5 \pm 0.630 \mathrm{SE} \\
(n=35)\end{array}$ & $\begin{array}{l}102.96 \pm 0.552 \mathrm{SE} \\
(n=35)\end{array}$ & $\begin{array}{l}103.93 \pm 0.828 \mathrm{SE} \\
(n=35)\end{array}$ & $\begin{array}{l}102.93 \pm 0.854 \mathrm{SE} \\
(n=35)\end{array}$ \\
\hline Osmolality (mmol/kg) & $\begin{array}{l}280.67 \pm 0.983 \mathrm{SE} \\
(n=35)\end{array}$ & $\begin{array}{l}280.06 \pm 1.168 \mathrm{SE} \\
(n=35)\end{array}$ & $\begin{array}{l}281.83 \pm 1.465 \mathrm{SE} \\
(n=35)\end{array}$ & $\begin{array}{l}283.50 \pm 2.204 \mathrm{SE} \\
(n=35)\end{array}$ & $\begin{array}{l}282.00 \pm 1.629 \mathrm{SE} \\
(n=34)\end{array}$ \\
\hline
\end{tabular}

Table 2 Group C (patients with repeated samplings): patients demographics and GCS score

\begin{tabular}{ll}
\hline & Group C (repeated sampling) \\
\hline$n$ & 23 \\
Age (mean) & $63.8 \pm 2.596 \mathrm{SE}$ \\
Age (max) & 80 \\
Age (min) & 27 \\
Male/female & $14 / 9$ \\
GCS $(n)$ & $\mathbf{G C S} \mathbf{1 4 - 1 5 / 9 - 1 3 / 3 - 8}$ \\
$6 \mathrm{~h}$ & $13 / 6 / 4$ \\
$48 \mathrm{~h}$ & $13 / 5 / 5$ \\
7 day & $15 / 4 / 4$ \\
\hline
\end{tabular}

GCS score 14-15 - mild TBI; GCS score 9-13 - moderate TBI; GCS 3-8 - severe TBI

test (Fig. 3). A significant increase is obvious in group C-6 h (mean 613.463 $\pm 49.055 \mathrm{SE}, n=26$ ) when compared to group A (controls) (mean $441.441 \pm 22.572 \mathrm{SE}, n=31$ ) and group B (mean 825.606 $\pm 23.690 \mathrm{SE}, n=30$ ). Group C-6 h also displays higher SP levels when compared to group C-48 h (mean 587.576 $\pm 48.363 \mathrm{SE}, n=26$ ). A significant increase is also present when comparing group C-7 day (mean 620.083 $\pm 46.743 \mathrm{SE}, n=27$ ) and group A. Even in the absence of visible brain contusions, a significant increase in SP is demonstrated when comparing group B (TBI with no contusions) and group A.

\section{S100B}

On one-way ANOVA test, there was a significant effect of TBI on S100B levels among different groups- $[F(4$, $95)=4.959, p=0.0011]$, post-hoc Tukey-Kramer method test (Fig. 4). A significant increase takes place in the first $6 \mathrm{~h}$ post-TBI when compared to controls [group C-6 h (mean $95.668 \pm 14.102 \mathrm{SE}, n=22$ ) vs. group A (mean $30.187 \pm 3.347 \mathrm{SE}, n=31$ )], followed by progression to baseline values in the next 7 days. S100B is also significantly increased in the presence of post-traumatic brain 


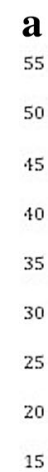

55

45

35

20 b 55

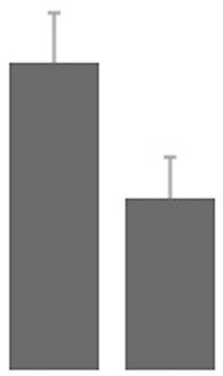

Fig. 1 a Response to TBI concerning NPY (pg/mL). Group A (Controls) - light grey; Group B (TBI) - grey; Group C (TBI with contusions; 6h, 48h and 7 days consecutively) - dark grey. Significant higher levels of NPY in group C-6h (mean 45.997 +/- 4.968 SE) and C-7d (mean 43.268 +/- 6.260 SE) when compared to group A (controls) (mean $19.702+/-1.462 \mathrm{SE}$ ), group B (TBI with no contusions) (mean $29.567+/-5.427 \mathrm{SE}$ ) and group C-48h (mean 32.395

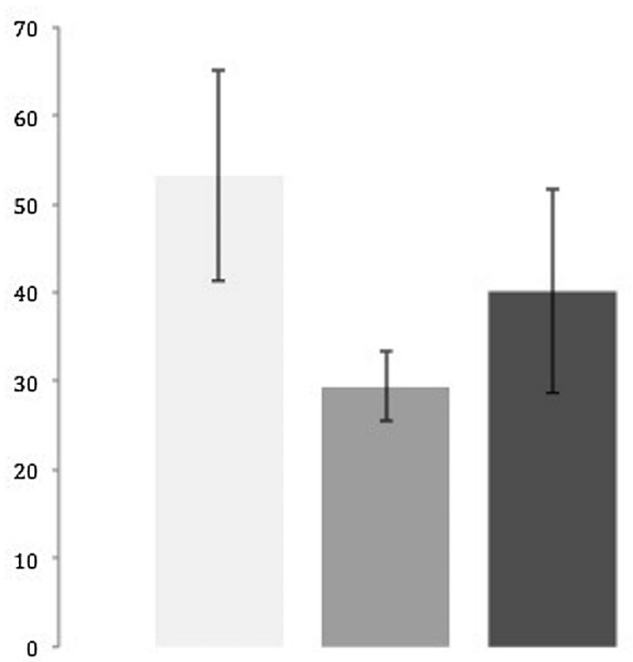

Fig. 2 Response to TBI concerning NPY, group C-6h, according to initial GCS scores (pg/mL). Mild TBI (light grey) (GCS 14-15), mean 53,210 +/- 11.910 SE; moderate TBI (grey) (GCS 9-13), mean $29.460+/-3.950$ SE; severe TBI (dark grey) (GCS 3-8), mean 40.114 +/- 11.435 SE. Stated differences did not reach statistical significance

contusions when compared to TBI with no contusions (both at $6 \mathrm{~h}$ ) [group C-6 h vs. group B (mean $42.303 \pm 6.302 \mathrm{SE}$, $n=29)$ ].

\section{Clinical laboratory tests}

On one-way ANOVA test, there was a significant effect of TBI on Magnesium levels among different groups- $[F(4$, $145)=5.682, p<0.001]$, post-hoc Tukey-Kramer method test (Fig. 5). Significant hypomagnesemia is present when comparing victims of TBI with brain contusions at $6 \mathrm{~h}$ and

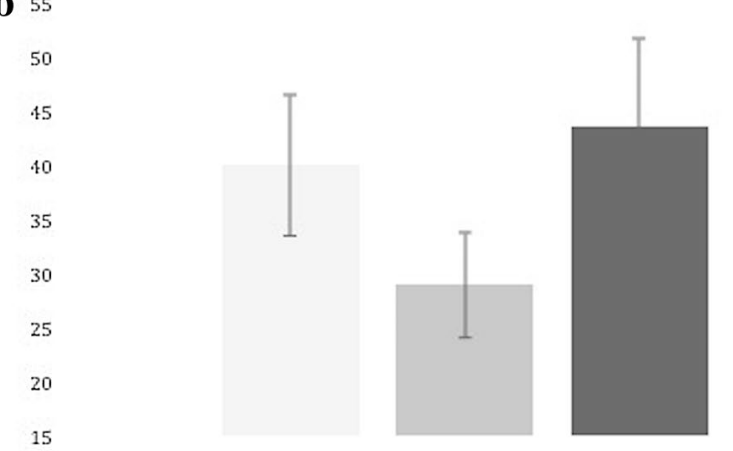

+/- 4.056 SE). b Response to TBI concerning NPY, repeated sampling (pg/mL). 6h post-TBI - light grey; 48 hours post-TBI - grey; 7 days post-TBI - dark grey. Similar pattern when comparing distinct timings in the same patient: group C-6h - mean 39.924 +/- 6.487 SE vs. group C-48h - mean $28.929+/-4.867 \mathrm{SE}$ vs. group C-7d - mean $43.467+/-8.072$ SE. Stated differences did not reach statistical significance

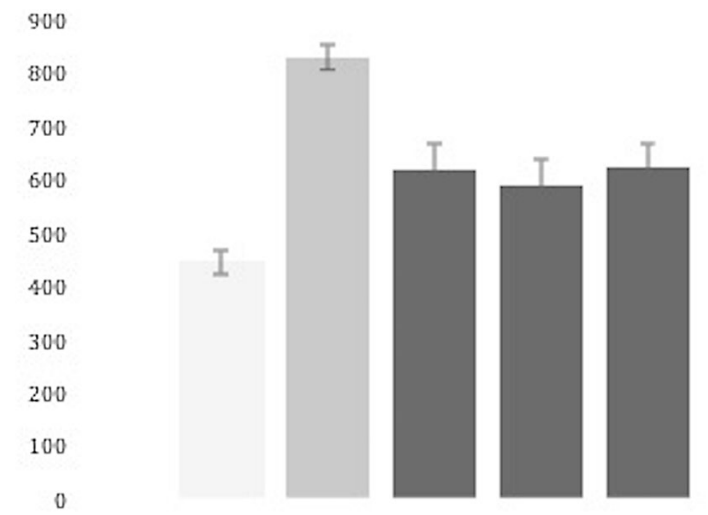

Fig. 3 Response to TBI concerning SP (pg/mL). Group A (Controls) - light grey; Group B (TBI) - grey; Group C (TBI with contusions; 6h, 48h and 7 days consecutively) - dark grey. Significant increase in group B (TBI with no contusions) (mean 825.606 +/- 23.690 SE) and group C-6h (mean $613.463+/-49.055 \mathrm{SE}$ ) when compared to group A (controls) (mean $441.441+/-22.572 \mathrm{SE})$

controls [group C-6 h (mean $0.754 \pm 0.015 \mathrm{SE}, n=33$ ) vs. group A (mean $0.897 \pm 0.021 \mathrm{SE}, n=35$ )] and when comparing different timings in all groups of TBI with brain contusions [group C-6 h vs. group C- $48 \mathrm{~h}$ (mean $0.811 \pm 0.019$ $\mathrm{SE}, n=34$ ) vs. group C-7 day (mean $0.925 \pm 0.039 \mathrm{SE}$, $n=34)$ ], with progressive recovery of $\mathrm{Mg}$ levels following TBI. Mean levels of $\mathrm{Mg}$ are also lower when comparing victims of TBI with and without brain contusions (at $6 \mathrm{~h}$ ) [group C-6 h vs. group B (mean $0.861 \pm 0.039 \mathrm{SE}, n=29$ )].

Mean values of Sodium, Potassium and Osmolality were unremarkable in what concerns different groups.

On one-way ANOVA test, there was a significant effect of TBI on Calcium levels among different groups- $[F(4$, 


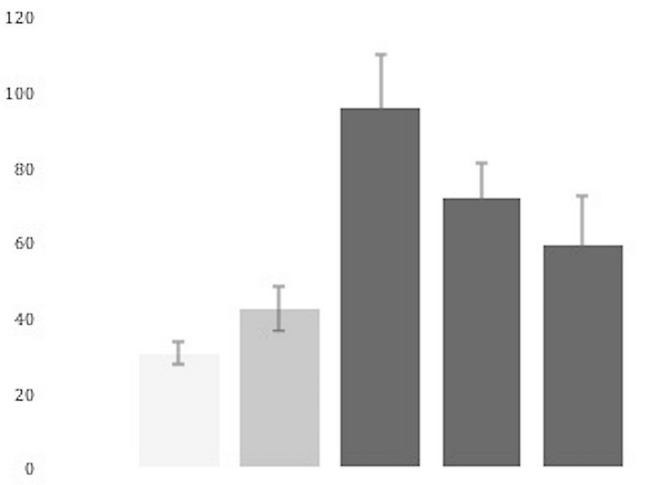

Fig. 4 Response to TBI concerning S100B (pg/mL). Group A (Controls) - light grey; Group B (TBI) - grey; Group C (TBI with contusions; $6 \mathrm{~h}, 48 \mathrm{~h}$ and 7 days consecutively) - dark grey. Significant increase in group C-6h (mean $95.668+/-14.102 \mathrm{SE}$ ) when compared to group A (controls) (mean $30.187+/-3.347 \mathrm{SE}$ ) and group B (TBI with no contusions) (mean $42.303+/-6.302 \mathrm{SE}$ )

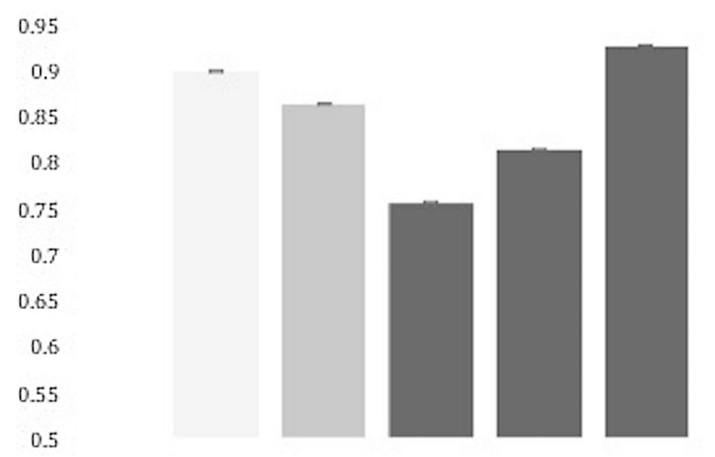

Fig. 5 Response to TBI concerning Magnesium (mmol/L). Group A (Controls) - light grey; Group B (TBI) - grey; Group C (TBI with contusions; $6 \mathrm{~h}, 48 \mathrm{~h}$ and 7 days consecutively) - dark grey. Significant hypomagnesemia in group C-6h (mean $0.754+/-0.015 \mathrm{SE}$ ) when compared to group A (controls) (mean $0.897+/-0.021 \mathrm{SE}$ ) and group B (TBI with no contusions) (mean $0.861+/-0.039$ SE). Magnesium levels progressively recover to their baseline levels at $48 \mathrm{~h}$ (group C-48h) (mean $0.811+/-0.019 \mathrm{SE}$ ) and 7 days (group C-7d) (mean $0.925+/-0.039 \mathrm{SE})$

146) $=9.593, p<0.001]$, post-hoc Tukey-Kramer method test (Fig. 6). A significant decrease is obvious when comparing controls and TBI with no brain contusions [group A (mean $9.46 \pm 0.063 \mathrm{SE}, n=35)$ vs. group B $(9.10 \pm 0.102 \mathrm{SE}$, $n=35)$ ], controls vs. TBI with brain contusions at $6 \mathrm{~h}$ [group A vs. group C-6 h (mean $8.73 \pm 0.149 \mathrm{SE}, n=35)$ ] and TBI with no brain contusions vs. TBI with brain contusions (both at $6 \mathrm{~h}$ ) (group B vs. group C-6 h).

On one-way ANOVA test, there was a significant effect of TBI on CRP levels- $[F(4,143)=16.056, p<0.001]$, post-hoc Tukey-Kramer method test (Fig. 7). A significant increase takes place when comparing TBI with brain contusions at $48 \mathrm{~h}$ (mean $7.706 \pm 1.106 \mathrm{SE}, n=35)$, TBI with

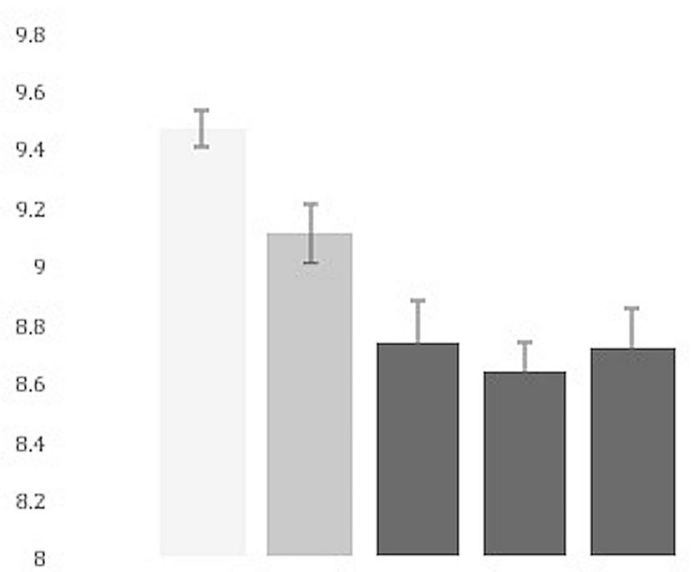

Fig. 6 Response to TBI concerning total serum Calcium (mg/dL). Group A (Controls) - light grey; Group B (TBI) - grey; Group C (TBI with contusions; $6 \mathrm{~h}, 48 \mathrm{~h}$ and 7 days consecutively) - dark grey. Significant hypocalcemia in group C-6h (mean $8.73+/-0.149 \mathrm{SE}$ ) when compared to group A (controls) (mean $9.46+/-0.063 \mathrm{SE}$ ) and group B (TBI with no contusions) (mean 9.10 +/- 0.102 SE)

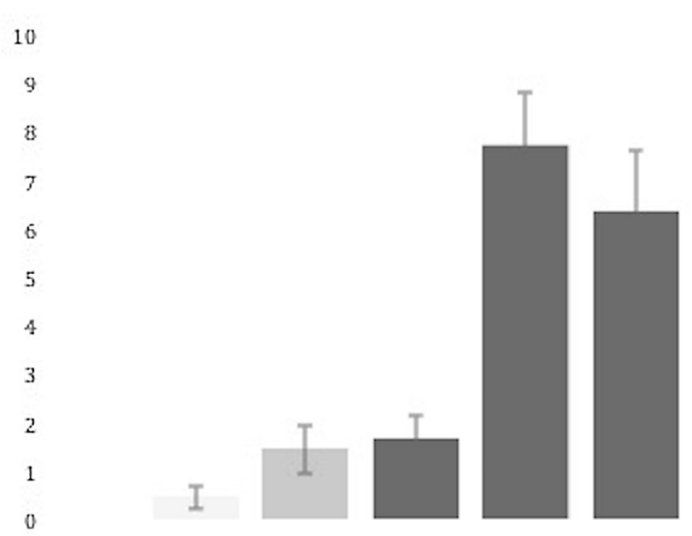

Fig. 7 Response to TBI concerning CRP (mg/L). Group A (Controls) - light grey; Group B (TBI) - grey; Group C (TBI with contusions; $6 \mathrm{~h}, 48 \mathrm{~h}$ and 7 days consecutively) - dark grey. Significant increase in CRP in relation to TBI and its different timings: group C-48h (mean 7.706 +/- 1.106 SE) vs. group C-6h (mean 1.674 +/- $0.469 \mathrm{SE})$ vs. group A (controls) (mean $0.461+/-0.244 \mathrm{SE}$ )

brain contusions at $6 \mathrm{~h}$ (mean $1.674 \pm 0.469 \mathrm{SE}, n=35)$ and controls (mean $0.461 \pm 0.244 \mathrm{SE}, n=35$ ) (Fig. 7).

\section{Discussion}

Despite growing interest in TBI's long-term consequences [34], several therapeutic protocols failed the test of facing modern evidence-based medicine [35]. Hypomagnesemia and SP's increment were tested as therapeutic targets [22], using specific antagonists (N-acetil-triptofan, canabinoid receptor-2 agonists) [35], with no clear benefits. 
We hypothesized that TBI leads to a multi-staged neuropeptide response, with an immediate response concerning SP, followed by compensatory NPY up-regulation. This response is divided, based on previous literature and according to our working model, now confirmed by the previously depicted results, in three different moments (Fig. 8):

- A hyper acute response exacerbated by SP, as an inflammatory response in the first hours following TBI, promoting cerebral vasogenic edema and neuroinflammatory processes;

- -An acute response determined by excitotoxic phenomena, partially mediated by Substance $P$, and a peak in S100B levels as a sign of neuronal/glial disturbance, progressing cerebral edema and neurological impairment;

- Finally, a delayed response with significant increase in NPY levels (and possibly others) as reinforcement to neuroprotective pathways, attenuating excitotoxicity and neuroinflammatory phenomena, with ancillary progressive recovery in Mg levels.

As depicted in the "Results", evidence of an early and delayed neuropeptide response to TBI is, therefore, shown. These findings are in line with the perceived role for neuropeptides and neurogenic inflammation as key components of post-TBI inflammation, along with SP's role in aspects of classical inflammatory response (activation of microglia/ astrocytes, leukocyte migration, degranulation of mast cells)
[20]. A minor initial increase in NPY was followed by an expected and significant decrease at $48 \mathrm{~h}$ post-TBI (coinciding with the usual timing for peak clinical deterioration and known deleterious secondary injury on a celular level) [36-38].

To better assess the relevance and adequacy of our working model, namely regarding NPY, this research protocol contemplated the possibility of comparing distinct patients among different groups (control, TBI, TBI with brain lesions in different timings) and, on a specific sub-group, compare NPY's levels in the same patient upon different timings. Similar trends in NPY's fluctuating levels were confirmed in both contexts, although an inferior number of patients in the paired-samples (repeated measurements) analysis might have prevented it from reaching statistical significance. This, in our opinion, reinforces our conclusions, as the statistical relevance of comparing specific, separate groups of patients is supplemented by the notion of biological continuity, considering the same objective response in the subset of patients assessed upon different timings. Assessment of NPY levels according to initial GCS scores did not provide any additional information, a fact most likely related to significant clinical variability upon initial presentation and small-sized groups.

This staged neuropeptide response is also in agreement with the well-known timings in brain injury and its biomarkers, with well-described post-traumatic hypomagnesemia and S100B levels peaking in the first $48 \mathrm{~h}$ post-TBI and
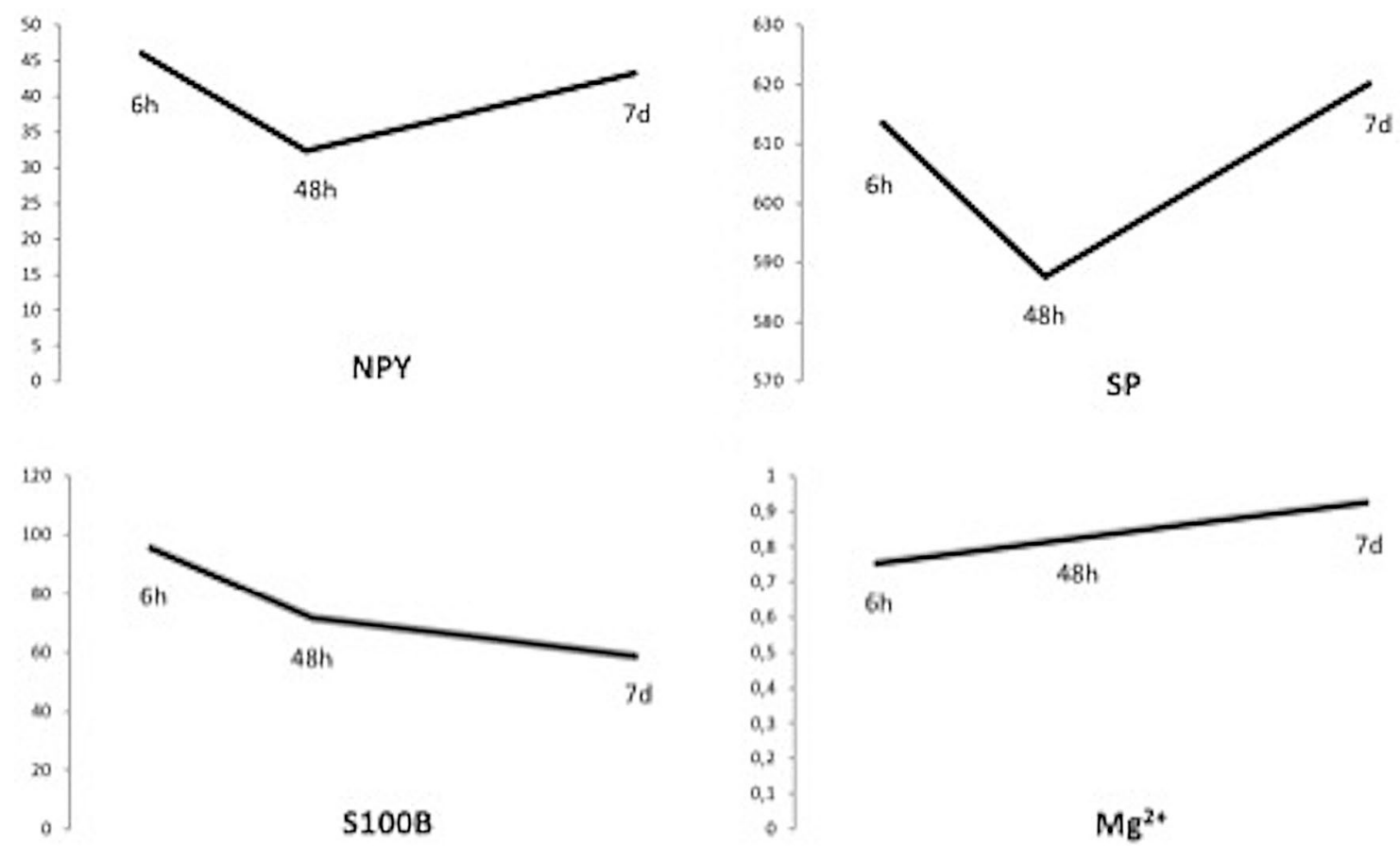

Fig. 8 Schematic representation of multi-staged response to TBI, with different timings for each element involved 
subsequently normalizing (assuming stabilization of the clinical picture and no ongoing parenchymatous lesions) [39]. Other aspects of SP's role in TBI still require better clarification, including the mechanisms behind its interference with BBB (some authors speculated on decreased endotelial expression of ZO-1 and claudin-5) [40].

Substance P levels in group B (TBI with no intracranial lesions) are increased when compared to controls (group A), an interesting finding that reinforces the relevance of TBI-related deleterious phenomena even in CT-negative patients. Rather counterintuitively, SP's levels are also further increased in group B when compared to group C, in which brain contusions are present. This fact, never reported before, can only be explained if considering a scenario on which neuroinflammation pathways upon TBI are surpassed by more relevant and disrupting events, including cell death and haemorrhages, in the context of direct brain injury with significant contusions. This would preclude the expected, but in this case impaired, neuroinflammation response, somewhat dependent on a more intact underlying brain structure. On the other hand, in groups $\mathrm{C}$, an expected decrease at $48 \mathrm{~h}$ was followed by a increase in SP's levels at 7 days, unlike the typical pattern described in the literature [15]. This fact, never reported before, is of uncertain significance and should be better elucidated in future studies.

Other findings, although of undeniable clinical relevance, are somewhat expected and deemed not relevant in this context. An obvious post-traumatic hypocalcemia is present, most likely in relation to well-described iatrogenic hemodilutional mechanisms [41], as expected in patients subjected to Neurointensive Care protocols, sometimes with aggressive fluid management. Significantly high CRP levels were also present in TBI patients. Despite several authors and research teams exploring the possibility of using CRP as a biomarker for TBI [42], this possibility, beyond the scope of this work, does not seem valid or useful [43], given CRP's heterogeneity, clinical ubiquity and lack of specificity in complex patients as in polytrauma.

Although other neurotransmitters are likely to be involved in post-traumatic neurogenic inflammation, namely CGRP (which might potentiate SP's action) [20], this research project focused on SP and NPY, as the most ubiquitous and potent neuropeptides [44]. NPY takes part, via five different receptor types in several different biological processes and events [24], including feeding stimulus and obesity [45], stress modulation [46], homeostatic balance, sleep, olfaction perception [47], cyrcadian rythms and endocrinologic disturbances [48]. Recent reports mention a possible role for NPY in neuroprotective strategies regarding psychiatric diseases [49], Alzheimer's disease and Parkinson's disease [50, 51], along with possible roles in modulating neuropathic pain and behavioral changes in temporal lobe epilepsy [24]. Neuropeptide Y, as well as its selective NPY Y2 receptor agonist
(NPY13-36), is known to act as an endogenous anticonvulsant by modulating glutamatergic hippocampal excitability [25], while displaying inhibitory action on cytotoxic cellular edema, by regulating $\mathrm{KCl}$-evoked glutamate release [52]. Interestingly, NPY is co-localized with SP on GABAergic interneurons and A1 neurons in supra-optic nuclei (despite different ATP-stimulated actions on vasopressin and oxytocine release) [53], among other organs [54], with some authors suggesting that NPY might counterbalance SP's action, namely on nociceptive pathways and/or inflammation $[55,56]$.

Given all evidence pointing to a neuroprotective action of NPY in different contexts, it is plausible to consider a key role for NPY in brain's response to TBI, a field of knowledge where the gap is tremendous. Sporadic reports have shown elevated plasmatic and serum NPY levels in animal models of trauma and humans [57, 58]. Long-term changes in hippocampal NPY expression are related to occurrence of post-traumatic epilepsy in animal models of TBI [59]. Not surprisingly, brain NPY levels and function are reduced in the elderly [60], a significant group in TBI, reinforcing the importance of potentiating this response. As previously mentioned, NPY is known to act as an antagonist to SP's and other neuropeptides activity, inhibiting SP release with anti-hyperalgesic effect via Y1 receptor signalling in the dorsal horn [55]. Therefore, reinforcing NPY's response is a potential therapeutic strategy, possibly along with SP modulation [14], in Neurotrauma, considering previously mentioned NPY's pro-neurogenic, pro-migratory and neuroprotective properties [61]. Neuropeptide Y supplementation protocols (namely by intranasal delivery) are underway in phase II/phase III clinical trials concerning other clinical contexts $[62,63]$.

Some issues can be raised concerning this research protocol. First, CT scans were initially classified by six experienced radiologists. Although a possible source of bias, it is unlikely that significant errors might arise from a simple assessment on having or not brain contusions/hematomas, an objective and rather obvious finding in scans. The presence of intraparenchymal lesions was the only variable (concerning NPY/SP/S100B and the presence or not of brain contusions in TBI victims, as shown in this study) - the number and classification of those lesions was not considered for the purpose of this study. Variations in size and severity of those lesions should influence neuropeptide response, but our intention was to demonstrate an encompassing phenomenon, regardless of severity. Likewise, it was not intended to assess a possible relation between neuropeptide response, clinical status and outcome.

Reported variability of SP and NPY serum and plasma levels is another possible bias [64]. Distinct sample preparation, qualitative differences in reagents, distinct analytical methods and SP's plasma/serum free and bound states 
could lead to wrong estimates $[64,65]$. Besides difficulties in dealing with complex patients in a complex environment (lost or mixed samples, change of clinical status), there was significant difficulty in obtaining valid results concerning SP's and S100B, with missed samples and outliers explaining most discrepancies in groups size concerning results.

As previously mentioned, none of the measured proteins is brain specific. Given this, as the traumatic event is well identified and time specific, any significant changes in protein's levels should be a TBI's direct consequence and valid extrapolations can be made. On the other hand, determination of neuropeptide's plasma levels is technically difficult and, as some issues with outliers were present, a tendency to spurious results should be kept in mind.

The decision on different timings for blood sampling was based on clinical grounds and previous research. Post-traumatic vasogenic edema peaks around the 3rd day post-TBI, unlike immediate cytotoxic edema [66]. A 48-h time point seems suitable for an adequate mid-asessment; while the 7-day time point provides, in our opinion, a good notion on a longer term. Concerning blood sampling until $6 \mathrm{~h}$ post-TBI, one should not ignore rapid fluctuations in SP's levels-dividing data into sub-groups (e.g., $30 \mathrm{~min}$ vs. $2 \mathrm{~h}$ vs. $6 \mathrm{~h}$ ) should provide additional and useful information. Given this, it was decided to keep the $6 \mathrm{~h}$ threshold, again based on clinical reasons: as in most terciary hospitals, most patients arrive the Emergency Department several hours after initial trauma; as all doctors know, patient and family reports are seldom reliable; it would be relatively impractical to repeatedly collect blood samples in a trauma patient in such a narrow time frame. Significantly, our data display significant changes at $6 \mathrm{~h}$ post-TBI, assuming an eventual underrepresentation. The decision on not taking additional blood samples in group B (at $48 \mathrm{~h}$ and 7 days following TBI) was based on clinical reasons, considering the nature of this study, the early discharges (as no traumatic findings were present) and the unnecessary blood sampling.

\section{Conclusion}

A multi-staged neuropeptide response to TBI is demonstrated. Future experimental studies (including animal models of trauma) should further characterize this response and eventually assess its potential as a therapeutic target, aiming at maximum functional recovery.

Acknowledgements Dra. Eulália Costa, for the technical support.

Funding No funding was received for this research.

Availability of data Supporting data can be accessed upon request.

\section{Compliance with ethical standards}

Conflict of interest None.

Ethical approval All procedures performed in studies involving human participants were in accordance with the ethical standards of the Institutional Research Committee (Centro Hospitalar e Universitário de Coimbra) and with the 1964 Helsinki Declaration and its later amendments or comparable ethical standards.

Informed consent Informed consent was obtained from all individual participants included in the study.

Consent for publication All authors have consented for the publication of this work.

\section{References}

1. Fu T, Jing R, McFaull S, Cusimano M. Health \& economic burden of traumatic brain injury in the emergency department. Can J Neurol Sci. 2016;43(2):238-47.

2. Humphreys I, Wood R, Phillips C, Macey S. The costs of traumatic brain injury: a literature review. Clin Outcomes Res. 2013;26(5):281-7.

3. Sorby-Adams AJ, Marcoionni AM, Dempsey ER, Woenig JA, Turner RJ. The role of neurogenic inflammation in blood-brain disruption and development of cerebral oedema following acute central nervous system injury. Int J Mol Sci. 2017;18(8).

4. DeKosky ST, Asken BM. Injury cascades in TBI-related neurodegeneration. Brain Inj. 2017;31(9):1177-82.

5. Shandra O, Winemiller A, Heithoff B, et al. Repetitive diffuse mild traumatic brain injury causes an atypical astrocyte response and spontaneous recurrent seizures. J Neurosci. 2019;39(10):1944-63.

6. Dobrachinski F, Gerbatin R, Sartori G, et al. Regulation of mitochondrial function and glutamatergic system are the target of guanosine effect in traumatic brain injury. J Neurotrauma. 2017;34(7):1318-28.

7. Djordevic J, Sabbir MG, Albensi BC. Traumatic brain injury as a risk factor for alzheimer's disease: is inflammatory signaling a key player? Curr Alzheimer Res. 2016;13(7):730-8.

8. Mouzon B, Chaytow H, Crynen G, et al. Repetitive mild traumatic brain injury in a mouse model produces learning and memory deficits accompanied by histological changes. J Neurotrauma. 2012;29(18):2761-73.

9. Croall I, Smith FE, Blamire AM. Magnetic resonance spectroscopy for traumatic brain injury. Top Magn Reson Imaging. 2015;24(5):267-74.

10. Stovell MG, Yan JL, Sleigh A, et al. Assessing metabolism and injury in acute human traumatic brain injury with magnetic resonance spectroscopy: current and future applications. Front Neurol. 2017;12(8):426.

11. Hoffman JR, Zuckerman A, Ram O, et al. Behavioral and inflammatory response in animals exposed to a low-pressure blast wave and supplemented with B-alanine. Aminoacids. 2017;49(5):871-86

12. Prakash R, Carmichael ST. Blood-brain barrier breakdown and neovascularization processes after stroke and traumatic brain injury. Curr Opin Neurol. 2015;28:556-64.

13. Minkkinen M, Iverson GL, Kotilainen AK, et al. Prospective validation of the scandinavian guidelines for initial management of minimal, mild, and moderate head injuries in adults. J Neurotrauma. 2019;36(20):2904-12. 
14. Lorente L, Martín M, Almeida T, et al. Serum substance P levels are associated with severity and mortality in patients with severe traumatic brain injury. Crit Care. 2015;19:192.

15. Vink R, Den Heuvel C. Substance P antagonists as a therapeutic approach to improving outcome following traumatic brain injury. Neurotherapeutics. 2010;7(1):74-80.

16. Lukacs M, Tajti J, Fulop F, Toldi J, Edvinsson L, Vecsei L. Migraine, neurogenic inflammation, drug development - pharmacochemical aspects. Curr Med Chem. 2017;24(33):3649-65.

17. Suvas $S$. Role of substance $P$ neuropeptide in inflammation, wound healing, and tissue homeostasis. J Immunol. 2017;199(5):1543-52.

18. Turner R, Vink R. The role of substance $P$ in ischaemic brain injury. Brain Sci. 2013;3(1):123-42.

19. Vink R, Gabrielian L, Thornton E. The role of substance P in secondary pathophysiology after traumatic brain injury. Front Neurol. 2017;8:304.

20. Corrigan F, Mander KA, Leonard AV, Vink R. Neurogenic inflammation after traumatic brain injury and its potentiation of classical inflammation. J Neuroinflamm. 2016;13(1):264.

21. Zhe Z, Hongyuan B, Wenjuan Q, Peng W, Xiaowei L, Yan G. Blockade of glutamate receptor ameliorates lipopolysaccharideinduced sepsis through regulation of neuropeptides. Biosci Rep. 2018;8(38):3.

22. Ameliorate JL, Ghabriel MN, Vink R. Magnesium enhances the beneficial effects of NK1 antagonist administration on blood-brain barrier permeability and motor outcome after traumatic brain injury. Magnes Res. 2017;30(3):88-97.

23. Ramamoorthy P, Wang Q, Whim M. Cell type-dependent trafficking of neuropeptide Y-containing dense core granules in CNS neurons. J Neurosci. 2011;31(41):14783-8.

24. Lynds R, Lyu C, Lyu GW, et al. Neuronal plasticity of trigeminal ganglia in mice following nerve injury. J Pain Res. 2017;9(10):349-57.

25. Silva AP, Xapelli S, Pinheiro PS, et al. Up-regulation of neuropeptide Y levels and modulation of glutamate release through neuropeptide $\mathrm{Y}$ receptors in the hippocampus of kainate-induced epileptic rats. J Neurochem. 2005;93(1):163-70.

26. Decressac M, Prestoz L, Veran J, Cantereau A, Jaber M, Gaillard A. Neuropeptide $Y$ stimulates proliferation, migration and differentiation of neural precursors from the subventricular zone in adult mice. Neurobiol Dis. 2009;34(3):441-9.

27. Geloso MC, Corvino V, Di Maria V, Marchese E, Michetti F. Cellular targets for neuropeptide Y-mediated control of adult neurogenesis. Front Cell Neurosci. 2015;16(9):85.

28. Spencer B, Potkar R, Metcalf J, et al. Systemic Central Nervous System (CNS)-targeted delivery of neuropeptide Y (NPY) reduces neurodegeneration and increases neural precursor cell proliferation in a mouse model of Alzheimer disease. J Biol Chem. 2016;291(4):1905-20.

29. Zhang Z, Ma Z, Zou W, Guo H, Liu M, Ma Y. The appropriate marker for astrocytes: comparing the distribution and expression of three astrocytic markers in different mouse cerebral regions. Biomed Res Int. 2019;24:9605265.

30. Thelin E, Nimer F, Frostell A, et al. A serum protein biomarker panel improves outcome prediction in human traumatic brain injury. J Neurotrauma. 2019;36(20):2850-62.

31. Frankel M, Fan L, Yeatts SD, et al. Association of very early serum levels of S100B, glial fibrillary acidic protein, ubiquitin C-terminal hydroxilase-L1 and spectrin breakdown roduct with outcome in proTECT III. J Neurotrauma. 2019;36(20):2863-71.

32. Mondello S, Sorinola A, Czeiter E, et al. Blood-based protein biomarkers for the management of traumatic brain injuries in adults presenting to emergency departments with mild brain injury: a living systematic review and meta-analysis. J Neurotrauma. 2018. https://doi.org/10.1089/neu.2017.5182.
33. Thelin E, Nelson D, Bellander BM. A review of the clinical utility of sérum S100B protein levels in the assessment of traumatic brain injury. Acta Neurochirur. 2017;159:209-25.

34. Lim L, Ho RM, Ho C. Dangers of mixed martial arts in the development of chronic traumatic encephalopathy. Int J Environ Res Public Health. 2019;16(2):254.

35. Donkin JJ, Cernak I, Blumbergs PC, Vink R. A substance P antagonist reduces axonal injury and improves neurologic outcome when administered up to $12 \mathrm{~h}$ after traumatic brain injury. J Neurotrauma. 2011;28:217-24.

36. Park $\mathrm{M}, \mathrm{Oh} \mathrm{H}$, Ko I, et al. Influence of mild traumatic brain injury during pediatric stage on short-term memory and hippocampal apoptosis in adult rats. J Exerc Rehabil. 2014;10(3):148-54.

37. Hellewell S, Semple B, Morganti-Kossmann M. Therapies negating neuroinflammation after brain trauma. Brain Res. 2016;1640:36-56.

38. Dikranian K, Cohen R, Macdonald C, et al. Mild traumatic brain injury to the infant mouse causes robust white matter axonal degeneration which precedes apoptotic death of cortical and thalamic neurons. Exp Neurol. 2008;211(2):551-60.

39. Ercole A, Thelin EP, Holst A, Bellander BM, Nelson D. Kinetic modelling of sérum S100B after traumatic brain injury. BMC Neurol. 2016;16:93.

40. Leonard AV, Manavis J, Blumbergs PC, Vink R. Changes in substance $\mathrm{P}$ and NK1 receptor immunohistochemistry following human spinal cord injury. Spinal Cord. 2014;52(1):17-23.

41. Giancarelli A, Birrer KL, Alban RF, Hobbs BP, Liu-deRyke X. Hypocalcemia in trauma patients receiving massive transfusion. J Surg Res. 2016;202(1):182-7.

42. Naghibi T, Mohajeri M, Dobakhti F. Inflammation and outcome in traumatic brain injury: does gender effect on survival and prognosis? J Clin Diagn Res. 2017;11(2):6-9.

43. Alves JL, Rato J, Silva V. Why does brain trauma research fail? World Neurosurg. 2019;130:115-21.

44. Sabban F, Vink R, Turner RJ. Inflammation in acute CNS injury: a focus on the role of substance P. Br J Pharmacol. 2016;173:703-15.

45. Zhang L, Bijker MS, Herzog H. The neuropeptide Y system: pathophysiological and therapeutic implications in obesity and câncer. Pharmacol Ther. 2011;131(1):91-113.

46. Sabban EL, Laukova M, Alaluf LG, Olsson E, Serova LI. Locus coeruleus response to single-prolonged stress and early intervention with intranasal neuropeptide Y. J Neurochem. 2015;135(5):975-86.

47. Kaniganti T, Deogade A, Maduskar A, Mukherjee A, Guru A, Subhedar N. Sensitivity of olfactory sensory neurons to food cues is tuned to nutritional states by neuropeptide $\mathrm{Y}$ signalling. BioRxiv. 2019. https://doi.org/10.1101/573170.

48. Morin LP. Neuroanatomy of the extended circadian rhythm system. Exp Neurol. 2013;243:4-20.

49. Morosawa S, Iritani S, Fujishiro H, et al. Neuropeptide Y neuronal network dysfunction in the frontal lobe of a genetic mouse model of schizophrenia. Neuropeptides. 2017;62:27-35.

50. Angelucci F, Gelfo F, Fiore M, et al. The effect of neuropeptide $\mathrm{Y}$ on cell survival and neurotrophin expression in in-vitro models of Alzheimer's disease. Can J Physiol Pharmacol. 2014;92(8):621-30.

51. Markaki E, Ellul J, Kefalopoulou Z, et al. The role of ghrelin, neuropeptide $\mathrm{Y}$ and leptin peptides in weight gain after deep brain stimulation for Parkinson's disease. Stereotact Funct Neurosurg. 2012;90(2):104-12.

52. Uckermann O, Wolf A, Franziska K, Wiedemann P, Reichenbach A, Bringmann A. Neuropeptide Y inhibits hypotonic glial cell swelling in the postischemic rat retina via glutamatergic Neuronto-Glia Signaling. Invest Ophtalmol Vis Sci. 2005;46:2224. 
53. Sampaolo S, Liguori G, Vittoria A, et al. First study on the peptidergic innervation of the brain superior sagittal sinus in humans. Neuropeptides. 2017;65:45-55.

54. Edyvane KA, Smet PJ, Trussell DC, Jonavicius J. Patterns of neuronal colocalisation of tyrosine hydroxylase, neuropeptide $\mathrm{Y}$, vasoactive intestinal polypeptide, calcitonin gene-related peptide and substance $\mathrm{P}$ in human uréter. J Auton Nerv Syst. 1994;48(3):241-55.

55. Nelson TS, Fu W, Donahue RR, et al. Facilitation of neuropathic pain by the NPY Y1 receptor-expressing subpopulation of excitatory interneurons in the dorsal horn. Sci Rep. 2019;10(9):7248.

56. Wang W, Xu T, Chen X, et al. NPY receptor 2 mediates NPY antidepressant effect in the mPFC of LPS rat by suppressing NLRP3 signaling pathway. Mediat Inflamm. 2019. https://doi. org/10.1155/2019/7898095.

57. He XY, Dan QQ, Wang F, et al. Protein network analysis of the serum and their functional implication in patients subjected to traumatic brain injury. Front Neurosci. 2019;12:1049.

58. McIntosh TK, Ferriero D. Changes in neuropeptide $\mathrm{Y}$ after experimental traumatic brain injury in the rat. J Cereb Blood Flow Metab. 1992;12(4):697-702.

59. Sun Z, Liu S, Kharmalov EA, Miler ER, Kelly KM. Hippocampal neuropeptide $\mathrm{Y}$ protein expression following controlled cortical impact and posttraumatic epilepsy. Epilepsy Behav. 2018;87:188-94.
60. Chiodera P, Volpi R, Pilla S, Cataldo S, Coiro V. Decline in circulating neuropeptide $\mathrm{Y}$ levels in normal elderly human subjects. Eur J Endocrinol. 2000;143:715-6.

61. Johnson V, Stewart J, Begbie F, Trojanowski J, Smith D, Stewart W. Inflammation and white matter degeneration persist for years after a single traumatic brain injury. Brain. 2013;136(1):28-422.

62. Fatoba O, Kloster E, Saft C, Gold R, Arning L, Ellrichmann G. L22 intranasal application of NPY and NPY13-36 ameliorate disease pathology in R6/2 mouse model of huntington's disease. J Neurol Neurosurg Psychiatry. 2016;87:A97-A9898.

63. Sabban EL, Serova LI. Potential of intranasal neuropeptide $Y$ (NPY) and/or melanocortin 4 receptor (MC4R) antagonists for preventing or treating PTSD. Mil Med. 2018;183(suppl1):408-12.

64. Campbell D, Raftery N, Tustin R, et al. Measurement of plasmaderived substance P: biological, methodological, and statistical considerations. Clin Vaccine Immunol. 2006;13(11):1197-203.

65. Corbally N, Powell D, Tipton KF. The binding of endogenous and exogenous substance-P in human plasma. Biochem Pharmacol. 1990;39:1161-6.

66. Szczygielski J, Glameanu C, Müller A, et al. Changes in posttraumatic brain edema in craniectomy-selective brain hypothermia model are associated with modulation of aquaporin-4 level. Front Neurol. 2018;9:799. 